ISSN: 2602-8506

Vol. 4, N¹., p.116-130 enero - marzo, 2020

Recibido: 06-11-2019/Aceptado: 09-12-2019/ Publicado: 04-01-2020

\title{
Alternativa de crecimiento económico en el cantón Chambo mediante el proyecto de factibilidad de la planta de enfriamiento de leche
}

\author{
Alternative for economic growth in the Chambo canton through the feasibility \\ project of the milk cooling plant
}

Edwin Rafael Oleas Carrillo. ${ }^{1}$, Luis Antonio Velasco Matveev. ${ }^{2}$, Julio Cesar Benavides Lara. ${ }^{3}$ \& María Augusta Burgos Mejía. ${ }^{4}$

\section{DOI: https://doi.org/10.33262/visionariodigital.v4i1.1067}

\begin{abstract}
Agricultural actions are a primary source of the Ecuadorian economy. In the canton Chambo, belonging to the province of Chimborazo, located $17 \mathrm{~km}$ from the city of Riobamba; agriculture, livestock, milk production, brick making and handmade tiles, are vital axes because they generate great impact on local and regional development.

With 65 families as dynamic partners, the San Antonio de Guayllabamba Agricultural Association is dedicated to the production and marketing of milk.

Consequently, the Ecuadorian industry is characterized by producing raw milk, which according to the ECB (2016) in the last year obtained a 3.5\% increase in daily production of liters, ranking the Sierra region as the most relevant in this food sector.

The present project seeks to evaluate the feasibility of a milk cooling plant as an alternative for economic growth in the San Antonio de Guayllabamba Association, so that the unit economic indicators allow highlighting the management of sustainable production and at the same time maintaining the economic, social, technological and environmental requirements, so this research is of an explanatory, hypothetical-deductive, historicallogical and synthetic-analytical nature in which direct observation and survey were data collection techniques.
\end{abstract}

1 Magister en Dirección de Empresas, Ingeniero Zootecnista, Docente de la Escuela Superior Politécnica de Chimborazo, eoleas@espoch.edu.ec

2 Magister en Gestión de Proyectos Socio Productivos, Docente de la Escuela Superior Politécnica de Chimborazo, lvelasco@espoch.edu.ec

3 Magister en Cadenas Productivas Agroindustriales, Ingeniero Zootecnista, Docente de la Escuela Superior Politécnica de Chimborazo, jbenavides@espoch.edu.ec

${ }^{4}$ Ingeniera en Finanzas, Jefa de Agencia Cooperativa de Ahorro CACPECO 
ISSN: 2602-8506

Vol. 4, N¹., p.116-130 enero - marzo, 2020

In the research process, Management and Business Development guidelines were considered, together with the Social Economy. In the main job search, it was determined that the milk cooling plant will help increase the economic income of the associates, thus generating a positive behavior of the producers towards the economic development that they wish to achieve in order to improve their quality of life.

Keywords: Feasibility, Cooling plant, Economic growth, Development, Social economy.

\section{Resumen}

Las acciones agropecuarias son una fuente primordial de la economía ecuatoriana. En el cantón Chambo, perteneciente a la provincia de Chimborazo, ubicado a $17 \mathrm{~km}$ de la ciudad de Riobamba; la agricultura, la ganadería, la producción de leche, la elaboración de ladrillos y tejas artesanalmente, son ejes de vital importancia pues generan gran impacto en el desarrollo local y regional.

Con 65 familias como socios dinámicos, la Asociación Agropecuaria San Antonio de Guayllabamba, se dedica a la producción y comercialización de leche.

La industria ecuatoriana se caracteriza por producir leche cruda, que según el BCE (2016) en el último año obtuvo un 3,5\% de incremento en la producción diaria de litros, catalogándose la región sierra como la más relevante de este sector alimenticio.

El presente proyecto busca evaluar la factibilidad de una planta de enfriamiento de leche como una alternativa de crecimiento económico en la Asociación San Antonio de Guayllabamba, de manera que los indicadores económicos unitarios permitan resaltar el manejo de una producción sostenible y al mismo tiempo se mantengan las exigencias económicas, sociales, tecnológicas y ambientales por lo que la presente investigación es de carácter explicativa, hipotética deductiva, histórica lógica y analítica sintética en la cual la observación directa y la encuesta fueron las técnicas de recolección de datos.

En el proceso de investigativo se consideró lineamientos de Gestión y Desarrollo Empresarial, conjuntamente con la economía social. En la principal búsqueda de trabajo se determinó que la planta de enfriamiento de leche ayudará a incrementar los ingresos económicos de los asociados, generando así un comportamiento positivo de los productores hacia el desarrollo económico que desean alcanzar para mejorar su calidad de vida.

Palabras clave: Factibilidad, Planta de enfriamiento, Crecimiento económico, Desarrollo Economía social.

\section{Introducción}

El consumo mundial de lácteos depende del aumento de la población con un $70 \%$ de la demanda se atribuye al crecimiento poblacional y el $30 \%$ sobrante al consumo por habitante de acuerdo a la Organización de la Naciones Unidas para la Agricultura y la Alimentación (FAO) pues el aumento de la población incrementa el consumo mundial de lácteos. 
Por sus grandes niveles de industrialización, poder adquisitivo, consumo per cápita y por su notable incremento poblacional el consumo de lácteos se ha concentrado en la mayoría de países donde existe estas condiciones y es por ello que las proyecciones del crecimiento promedio de la población mundial han generado un mayor dinamismo en términos relativos en los países industrializados y en los en vías de desarrollo en especial denominación a los países emergentes sobre temas de alimentación.

En la mayoría de países se ha considerado un mayor abasto de mismo, considerándose como una prioridad a nivel nacional e internacional, por tanto, se establecen políticas de protección en el sector en cuanto al consumo y producción de lácteos. Por otra parte, según datos del Banco Central del Ecuador (BCE), la producción de leche en países como Bolivia, Colombia, Ecuador y Perú alcanzaron durante el periodo del 2010 al 2015 un promedio de 16,152.000 TM lo que supone un crecimiento anual de 2,8\% superior a la tasa de crecimiento poblacional. La industria ecuatoriana se caracteriza por producir leche cruda, que según el BCE (2016) en el último año obtuvo un 3,5\% de incremento en la producción diaria de litros, catalogándose la región sierra como la más relevante de este sector alimenticio. De acuerdo a las tendencias de los mercados internacionales, la producción ecuatoriana muestra un alza, en volumen (litros) de leche producidos en cada unidad productiva, esto obedece a la alta tecnificación de los procesos y producción de derivados. Según la Asociación de Ganaderos de la Sierra y Oriente (AGSO) en la región sierra se registra una contribución del $73.5 \%$, por su parte en la región costa registra el $16.66 \%$ y el oriente aborda un promedio de $9.84 \%$ (AGSO, 2016).

La Asociación San Antonio de Guayllabamba, es una organización que se encuentra ubicada en la comunidad del mismo nombre en el cantón Chambo en la Provincia de Chimborazo, está constituida como una entidad de derecho privado sin fines de lucro y está orientada a potencializar la producción agrícola y pecuaria para el desarrollo comunitario, esta organización ha venido trabajando desde el año 2009. La asociación está conformada por 65 miembros, quienes se dedican a la ganadería; y mantiene una producción diaria promedio de 15000 litros de leche cruda en 165 hectáreas de terreno.

El desarrollo económico y social de sus integrantes se consolida como una prioridad de la entidad. No obstante, los principales problemas se deben al desconocimiento del impacto que su gestión ha tenido en el bienestar de la comunidad, ya que no cuenta con estrategias y acciones que se encaminen a elevar la calidad de vida de sus integrantes, esto ha conllevado a que su gestión no se consolide como un dinamizador de los factores sociales y económicos para mejorar el acceso a mejores niveles de vida de sus asociados. (SERRANO, 2005)

\section{Metodología}

Para el desarrollo de la investigación se trabajó bajo los parámetros de una investigación explicativa pues estuvo dirigida a encontrar las causas de los eventos, sucesos o fenómenos sociales, intentando establecer así la relación causa/efecto. En la fase del planteamiento del problema se empleó la investigación de carácter hipotético-deductivo, logrando así la formulación 
de una idea a defender inicial que sirvió de guía en el desarrollo del trabajo. Al hablar de crecimiento económico también lo hacemos de realidad y por ello para su comprensión se utilizó el análisis y la deducción de situaciones y conclusiones, así como de la historia y la lógica.

Los métodos que coadyuvaron en la consecución del objetivo principal fueron la: observación directa, el análisis documental y la encuesta. El enfoque de la propuesta es mixto, pues se pretendió realizar un análisis objetivo de la situación socioeconómica de los productores de leche de la Asociación San Antonio de Guayllabamba, así como la valoración de las percepciones y experiencias de los productores, a través de los datos recolectados.

En lo que tiene que ver a la población de estudio no se extrajo una muestra a que el universo conocido era de 65 socios activos a los cuales se les realizó un cuestionario que permitió conocer las causas del bajo desarrollo económico para posteriormente diseñar estrategias.

En cuanto a las variables se realizó la matriz de operacionalización la misma que contribuyó a la clarividencia de las definiciones e indicadores. Se formuló la hipótesis y para su comprobación se empleó el método del cálculo del Chi Cuadrado, esto se realizó con la información recopilada referente a los aspectos que categorizan el poder adquisitivo bajo el enfoque de desarrollo económico en dos escenarios a los productores: el primer contexto la situación actual, es decir sin el proyecto y sus ingresos económicos; en un segundo escenario, ya se cuenta con el proyecto y la generación de ingresos económicos.

Dentro del análisis situacional, se ejecutó un taller con todos los productores de la Asociación, con el fin de identificar las necesidades latentes de los mismos, se aplicó la técnica de construcción de la Matriz DAFO la misma que evidencia de manera óptima las debilidades, amenazas, fortalezas y oportunidades de la organización.

De manera similar se estudió el comportamiento de la oferta y la demanda, y se concluyó respectivamente que: los principales consumidores de leche en el cantón Chambo son aproximadamente once queseras artesanales locales que se encargan de recorrer el cantón recolectando la leche; la comunidad que más aporta con su nivel de producción es Guayllabamba con 7342 litros diarios a pesar de que no cuentan con muchos ejemplares productores de leche.

\section{Resultados}

Con el propósito de identificar la situación actual los productores integrantes de la Asociación San Antonio de Guayllabamba, se aplicó un cuestionario con interrogantes referentes a sus ingresos económicos y cada una de las aristas que se involucran, evidenciando así los siguientes resultados. (MONTUFAR, E. 2014).

El nivel de producción de leche de los asociados, el 69\% produce de 26 a 50 litros diarios, que se podría considerar como un con asesoramiento técnico nivel medio, a pesar de las dificultades que experimentan por no contar para realizarlo. 
ISSN: 2602-8506

Vol. 4, $\mathrm{N}^{\circ} 1 .$, p.116-130 enero - marzo, 2020

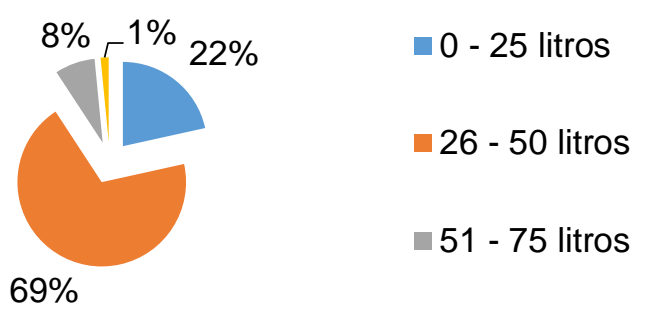

Figura 1. Nivel de producción de leche de los asociados Fuente: Elaboración propia.

Con respecto al precio de venta al público de cada litro lo investigado fue que el $39 \%$ lo comercializa a 0,25 centavos de dólar, mientras que el 35\% siguiente lo hace a 0,35 centavos de dólar, encontrando así la ausencia de coordinación en el establecimiento y fijación de precios, cayendo así en la infravaloración del producto y en ocasiones en la no recuperación de costos de producción. (CORAGGIO 2016).

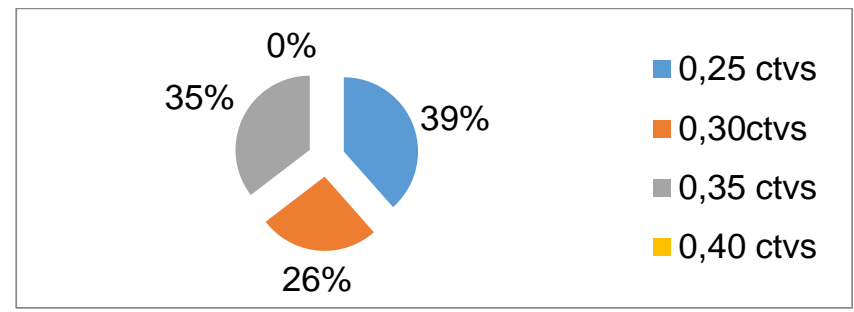

Figura 2. Precio de venta al público

Fuente: Elaboración propia.

En cuanto a si los ingresos provenientes de la venta de leche sin procesar cubren las necesidades básicas los productores, se identificó que el $83 \%$ de los socios no las cubre con la venta de su producción, debido a que no existe una política interna que regule y norme los precios de venta, por tanto, no todos los socios tienen un margen de venta estable sobre sus productos. (CORAGGIO 2016).

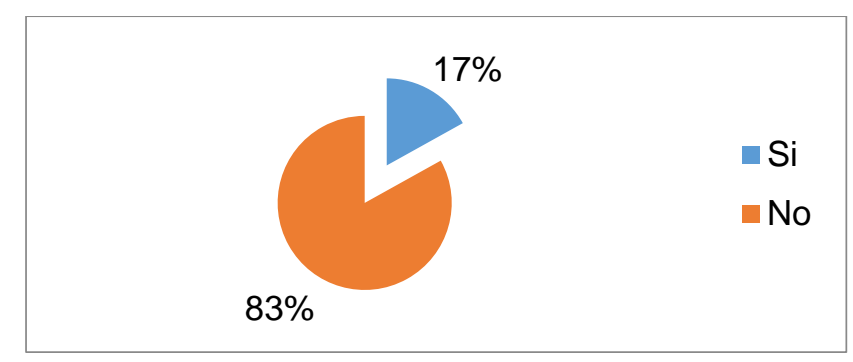

Figura 3. Venta de leche sin procesar

Fuente: Elaboración propia. 
ISSN: 2602-8506

Vol. 4, N¹., p.116-130 enero - marzo, 2020

Otra de las interrogantes planteadas, abordó la temática sobre si existe un centro de acopio y procesamiento de la leche para su venta, el $97 \%$ de los encuestados la desconocen, por tal motivo esto evidencia la importancia del proyecto debido a la necesidad de generar un alto valor agregado al producto, de la mano del cumplimiento de las normas de calidad y salubridad que los organismos de control exigen.

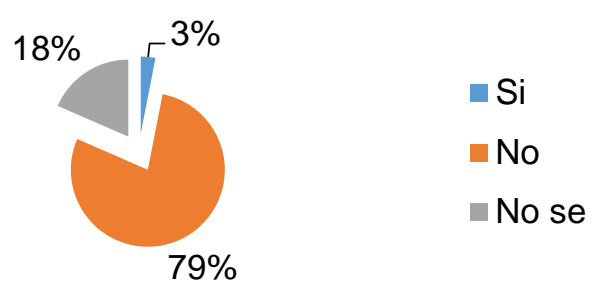

Figura 4. Existencia de centro de acopio y procesamiento de leche para su venta Fuente: Elaboración propia.

Una vez identificada esta variable, se planteó si los integrantes de la asociación estarían dispuestos a aportar con recursos humanos y económicos para crear una planta de enfriamiento de leche, obteniendo una respuesta del $58 \%$ que sólo lo harían con el factor humano, mientras que un reducido $25 \%$ que lo haría humana como económicamente.

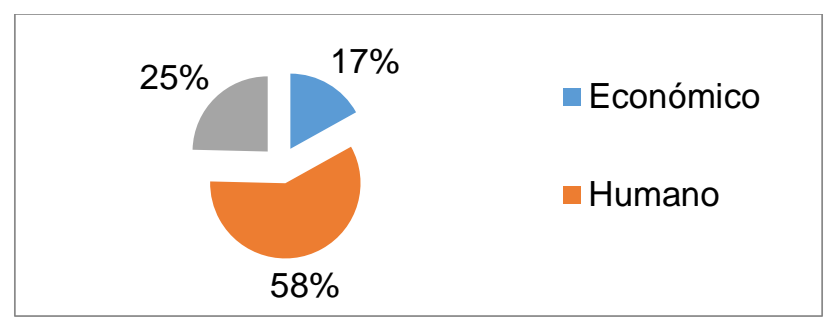

Figura 5. Presencia de planta de enfriamiento de leche Fuente: Elaboración propia.

No obstante, el $100 \%$ de los asociados manifestaron que estarían de acuerdo a participar en la creación de la planta de enfriamiento de leche, todos impulsados por la necesidad que la Asociación San Antonio posee de pertenecer a un proyecto que brinde apoyo a los productores de la localidad para mejorar el nivel de producción, calidad y ventas de sus productos, y asesore en la parte administrativa-estratégica de la organización.

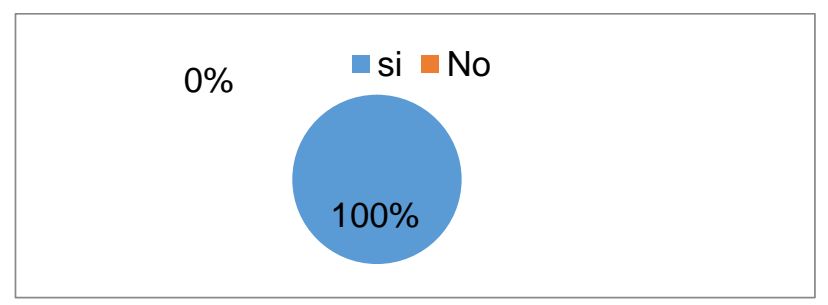

Figura 6. Participación de planta de enfriamiento de leche 
Fuente: Elaboración propia.

Consecuentemente, se indagó sobre si los productores consideran que la creación de la planta de enfriamiento de leche les ayudaría a incrementar sus ingresos económicos, ante lo cual están convencidos de que sí lo hará; pues dicho proyecto mejorará el proceso, y calidad del producto.

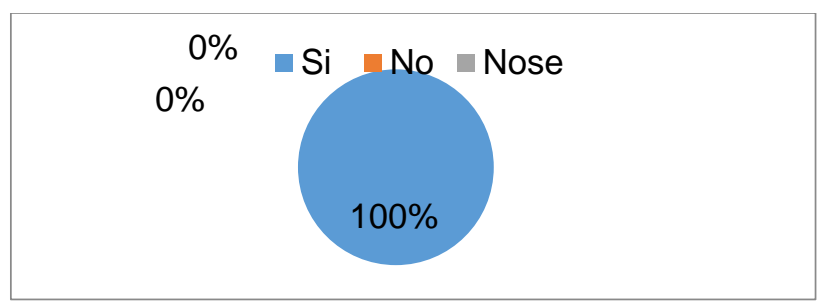

Figura 7. Necesidad de creación de una planta de enfriamiento de leche Fuente: Elaboración propia.

Con la información obtenida se ratifica la despectiva necesidad de que a través de un proyecto se cree una planta de enfriamiento de leche con el afán de establecer un agregado al valor constante a la producción de la Asociación San Antonio, quienes buscan mejorar su calidad de vida mediante el aumento de sus ingresos económicos para así satisfacer sus necesidades básicas y múltiples en diversos ámbitos.

\section{Discusión}

Mediante los datos obtenidos del estudio, y del análisis situacional a través de la Matriz DAFO y del análisis de la oferta y la demanda, se analizó la necesidad de definir estrategias que servirán de ayuda para la realización del presente proyecto

En el medio de la comercialización se establece la estrategia de trabajar con clientes directos, en este caso Nutri Leche, para que adquiera la leche producida por la asociación, con esta estrategia, los socios no tendrán que incurrir en gastos innecesarios al momento de poder expender su producción.

Dentro del primer año de los ingresos provenientes de las ventas ayudarán a mejorar y equipar el centro de acopio y enfriamiento con equipos altamente tecnificados para llevar a cabo la iniciación del procesamiento de los derivados lácteos y también para el aumento de la leche recolectada desde los sectores cercano al lugar, mejorando así la estrategia de venta.

Para la estrategia sobre precios, se fijará como referencia el precio que se paga de manera general en el sector, en el cual se considerará los gastos operacionales de acopio de leche, así como maquinaria a invertir y capital de trabajo, también, se estimará un precio final en el cual se incluya factores como nuevos segmentos de mercado y requerimientos de calidad.

Para la adquisición de la leche de entre los socios del predio se tomará como referencia los precios que rigen dentro del sector y se considerará la calidad de producto para subir o bajar el precio fijado, así como también la continuidad de entrega. 
Para fortalecer a la organización se prevé realizar talleres de capacitación a los socios con la finalidad de que todos sean partícipes en los procesos de toma de decisiones por parte del Comité de Gestión Comercial en temas relacionados a gestión administrativa de la asociación.

Dentro del proyecto se contará con el centro de acopio y enfriamiento de leche el mismo que contará con un silo, con una capacidad de almacenamiento veinte mil litros. La unidad de medida se expresará en litros y el tiempo se definirá en días. (Revilla, A. 1982).

En cuanto a la capacidad instalada, se prevé que 500 litros por cada hora sean alojados dentro del proceso de enfriamiento, datos especificados en relación a la capacidad de la maquinaria a instalar. En cuanto a los componentes que determinan el proyecto, se establece una serie de factores que sustentan la viabilidad técnica del mismo, entre ellos se encuentran los que se detallan a continuación:

- Disponibilidad de agua: posee con una red de agua potable que abastece al sector investigado.

- Suministros de energía: existe una red de cableado eléctrico, la misma que servirá para alimentar de energía eléctrica a los equipos y maquinarias que se han considerado en el proyecto.

- Cercanía de fuentes de abastecimiento.

- Cercanía del mercado: A pesar de que, en el proyecto considera un cliente principal, la asociación se encuentra cercana a más productores de derivados de la leche que pueden ser considerados como clientes potenciales.

La identificación del proceso se lo realizará por medio del enfriamiento por intercambiador de temperatura, debido a que este método es el más adecuado para grandes cantidades de líquidos; y consiste en enfriar la leche a través de un intercambiador de calor, en donde la temperatura es regulada por placas o tubos. Una vez enfriada la leche es transportada a un tanque de almacenamiento, el mismo que cuenta con aislamiento de poliuretano que permite mantener por 24 horas la temperatura deseada. Para este proceso, se debe contar con un Banco de Hielo, el mismo que sirve para enfriar el agua, posteriormente se transporta hacia el enfriador de placas.

Los pasos a seguir serán: recolección, muestreo, envío a laboratorio para el análisis correspondiente y en caso de cumplimiento de estándares la leche pasa a filtración, enfriamiento, almacenamiento, concluyendo en la carga, es decir la leche es conducida por tuberías desde los silos, hasta una bomba sanitaria, con el fin de expulsarla hasta los vehículos que servirán de transporte hacia el destino final del producto.

El proyecto también ha considerado, un inventario de equipos necesarios eventualmente acompañado por las especificaciones técnicas de cada uno que son necesarias para dar cumplimiento a los objetivos del proyecto. Entre ellos tenemos tanque recibo, filtro sanitario para leche, válvulas, bomba sanitaria de leche, unidad de frío, banco de hielo, caldera, bomba alimentadora de agua para caldera, silos, moto reductores.

Para el avance del proyecto se plantea la presente estructura organizativa con el fin de garantizar el desarrollo endógeno de la Asociación, su fundamentación se la realiza en base al Acuerdo No. 
ISSN: 2602-8506

Vol. 4, N¹., p.116-130 enero - marzo, 2020

16, mediante el cual la Dirección de Chimborazo del Ministerio de Agricultura, Ganadería, Acuacultura y Pesca otorga la personería jurídica a la Asociación.

La estructura de la empresa debe responder a criterios de eficacia y eficiencia, asociados a la gestión de la calidad y al trabajo en equipo, para alcanzar los resultados que demanda los clientes potenciales. (CABRA, C., \& DEL PILAR, S. C. 2007). En la cabeza se presentará la Junta General de Socios, Directorio, Gerente, secretaria y/o Contadora (personal auxiliar), Técnico de Producción o Laboratorio.

El layout de la planta de enfriamiento se presenta a continuación:

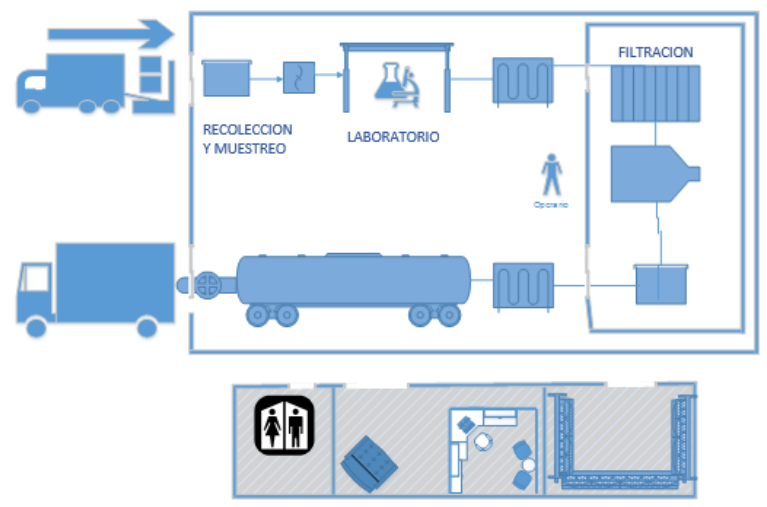

Figura 8. Planta de enfriamiento

Fuente: Elaboración propia.

El presente proyecto se establece una estrategia para el fortalecimiento de espacios agrícolas limpios y sustentables, con el fin de evitar grandes impactos ambientales negativos.

Tabla 1. Espacios agrícolas limpios y sustentables mediante una estrategia de fortalecimiento

\begin{tabular}{|c|c|c|c|}
\hline \multirow[t]{2}{*}{$\begin{array}{l}\text { Parámetros } \\
\text { ambientales }\end{array}$} & \multirow[t]{2}{*}{ Posibles efectos } & \multicolumn{2}{|c|}{$\begin{array}{l}\text { Impacto } \\
\text { ambiental }\end{array}$} \\
\hline & & + & - \\
\hline Agua & Contaminación del agua & & $\mathrm{x}$ \\
\hline Aire & Contaminación del aire & & $\mathrm{x}$ \\
\hline Suelo & Erosión del suelo & $\mathrm{x}$ & \\
\hline Usos del territorio & $\begin{array}{l}\text { El proyecto será ubicado en el terreno de la } \\
\text { Asociación San Antonio de Guayllabamba }\end{array}$ & $\mathrm{x}$ & \\
\hline Nivel Cultural & $\begin{array}{l}\text { Afianza el empoderamiento para mejorar la } \\
\text { conservación del medio ambiente }\end{array}$ & $\mathrm{X}$ & \\
\hline $\begin{array}{l}\text { Mejoramiento de } \\
\text { producción }\end{array}$ & Perfeccionamiento de procesos. & $\mathrm{x}$ & \\
\hline Infraestructura & Instalaciones fuera de áreas protegidas & $\mathrm{x}$ & \\
\hline
\end{tabular}

Fuente: Elaboración propia. 
ISSN: 2602-8506

Vol. 4, N¹., p.116-130 enero - marzo, 2020

Al tratarse de un proyecto con beneficios sociales se procedió a realizar el estudio financiero del mismo dando como resultados los siguientes:

Tabla 2. Análisis financiero

\begin{tabular}{lcc}
\hline \multicolumn{1}{c}{ Detalle } & Monto de inversión & $\begin{array}{c}\text { \% de } \\
\text { inversión }\end{array}$ \\
\hline Capital propio & $76.047,63$ & $100 \%$ \\
Capital financiado & 0 & $0 \%$ \\
TOTAL & $\$ 76.047,63$ & $100 \%$ \\
\hline
\end{tabular}

Fuente: Elaboración propia.

Como toda actividad genera egresos se ha proyectado para cinco años su futuro comportamiento.

Tabla 3. futuro con su proyección en 5 años

\begin{tabular}{|c|c|c|c|c|c|}
\hline \multirow{2}{*}{ CONCEPTO } & \multicolumn{5}{|c|}{ AÑNOS } \\
\hline & 1 & 2 & 3 & 4 & 5 \\
\hline EGRESOS VARIABLES & & & & & \\
\hline $\begin{array}{l}\text { COSTO DE VENTAS } \\
\text { Costo de venta de la leche } \\
\text { EGRESOS FIJOS } \\
\text { GASTOS } \\
\text { OPERACIONALES }\end{array}$ & 2052000,00 & 2158820,55 & 2271202,01 & 2389433,79 & 2513820,21 \\
\hline Gastos Administrativos & 24085,76 & 24482,70 & 24886,17 & 25296,30 & 25713,18 \\
\hline Gastos Operacionales & 446015,75 & 453366,09 & 460837,56 & 468432,17 & 476151,93 \\
\hline Depreciaciones & 3922,41 & 3922,41 & 3922,41 & 3406,25 & 3406,25 \\
\hline Amortizaciones & 400,00 & 400,00 & 400,00 & 400,00 & 400,00 \\
\hline GASTOS NO & & & & & \\
\hline OPERACIONALES & & & & & \\
\hline Gastos Financieros & 0,00 & 0,00 & 0,00 & 0,00 & 0,00 \\
\hline $\begin{array}{l}\text { Otros Gastos no } \\
\text { Operacionales }\end{array}$ & 0,00 & 0,00 & 0,00 & 0,00 & 0,00 \\
\hline EGRESO TOTAL & 2526423,93 & 2640991,75 & 2761248,16 & 2886968,50 & 3019491,56 \\
\hline
\end{tabular}

Fuente: Elaboración propia.

La tasa mínima aceptable de rentabilidad TMAR que la empresa deberá ganar para que sus socios o accionistas sobre el $100 \%$ de su aportación ganen un 8,73\%, menos de este porcentaje el negocio no es favorable. (ASSIDON 2002).

Para evaluar el proyecto se debe calcular el VAN, TIR, BC/C y Punto de Equilibrio, indicadores que estiman un comportamiento positivo para la comunidad y sus habitantes. 
ISSN: 2602-8506

www.visionariodigital.org

Vol. 4, N¹., p.116-130 enero - marzo, 2020

Tabla 4. Tasa mínima de rentabilidad - TMAR

\begin{tabular}{|c|c|c|c|c|c|c|}
\hline TMAR & \multicolumn{6}{|c|}{$8,73 \%$} \\
\hline AÑOS & 0 & 1 & 2 & 3 & 4 & 5 \\
\hline \multicolumn{7}{|l|}{ FLUJO NETOS } \\
\hline DE FONDOS & & & & & & \\
\hline DÓLARES & $(76.047,63)$ & $(8.926,13)$ & $10.580,31$ & $31.185,74$ & $52.681,81$ & $114.822,53$ \\
\hline $\begin{array}{c}\text { VALOR } \\
\text { ACTUAL NETO }\end{array}$ & \multicolumn{6}{|c|}{$\$ 62.227,04$} \\
\hline
\end{tabular}

Fuente: Elaboración propia.

El proyecto genera o va a rendir $\$ 62.227,04$ adicionales; es decir luego de haber recuperado lo mínimo esperado del 8,73\% por haber invertido en el proyecto; la inversión es aceptable.

Tabla 5. Tasa mínima de retorno - TIR

\begin{tabular}{ccccccc}
\hline $\begin{array}{c}\text { TMAR } \\
\text { AÑOS }\end{array}$ & $\mathbf{0}$ & $\mathbf{1}$ & $\mathbf{2}$ & $\mathbf{3}$ & $\mathbf{4}$ & $\mathbf{5}$ \\
\hline $\begin{array}{c}\text { FLUJO NETOS } \\
\text { DE FONDOS }\end{array}$ & $76.047,63$ & $8.926,13$ & $10.580,31$ & $31.185,74$ & $52.681,81$ & $114.822,53$ \\
TASA INTERNA & & & & & & \\
DEL RETORNO & & & $25 \%$ & & \\
\hline
\end{tabular}

Fuente: Elaboración propia.

La TIR del 25\%, comparada con la TMAR del 8,73\% es mayor, lo que implica que el proyecto en el tiempo de 5 años horizonte es aceptable, debido a que va a rendir un 16,27 puntos porcentual, más de lo mínimo que se esperaba para el mismo. (ASSIDON 2002). Siendo un proyecto rentable que generará ganancia a los socios.

En el proyecto que se presenta, se prevé que, por 1,00 USD invertido, este generará 1,18 USD, lo que indica que una vez recuperado 1,00 USD, se tiene obtiene como beneficio 0,18 ctvs.

Tabla 6. Beneficio/Costo

\begin{tabular}{|c|c|c|c|c|c|}
\hline & \multirow[t]{2}{*}{ INGRESOS } & \multirow[t]{2}{*}{ COSTOS } & $\begin{array}{c}\text { Coeficiente de } \\
\text { actualización }(1+ \\
\text { i) } n \\
\end{array}$ & \multirow{2}{*}{$\begin{array}{c}\text { Beneficio } \\
\text { Actualizado } \\
\text { (ingresos / } \\
\text { coeficiente }\end{array}$} & \multirow{2}{*}{$\begin{array}{c}\text { Costo } \\
\text { Actualizado } \\
\text { (egresos / } \\
\text { coeficiente }\end{array}$} \\
\hline & & & $\begin{array}{c}\mathbf{i}=\underset{8,73 \%}{\text { TMAR }}= \\
=\end{array}$ & & \\
\hline 0 & & $-76047,63$ & 1,00000000000000 & 0,00 & $76.047,63$ \\
\hline 1 & 2970000,00 & 2526423,93 & 1,08730000000000 & $2.731 .536,83$ & $2.323 .575,76$ \\
\hline 2 & 3124608,70 & 2640991,75 & 1,18222129000000 & $2.642 .998,16$ & $2.233 .923,36$ \\
\hline 3 & 3287265,83 & 2761248,16 & 1,28542920861700 & $2.557 .329,34$ & $2.148 .113,75$ \\
\hline 4 & 3458390,37 & 2886968,50 & 1,39764717852926 & $2.474 .437,34$ & $2.065 .591,77$ \\
\hline 5 & 3638423,10 & 3019491,56 & 1,51966177721487 & $2.394 .232,16$ & $1.986 .949,73$ \\
\hline & & & & $12.800 .533,83$ & $10.834 .202,00$ \\
\hline
\end{tabular}

$\mathrm{RB} / \mathrm{C}=1,18$

Fuente: Elaboración propia. 
El punto de equilibrio del proyecto se evidencia a continuación:

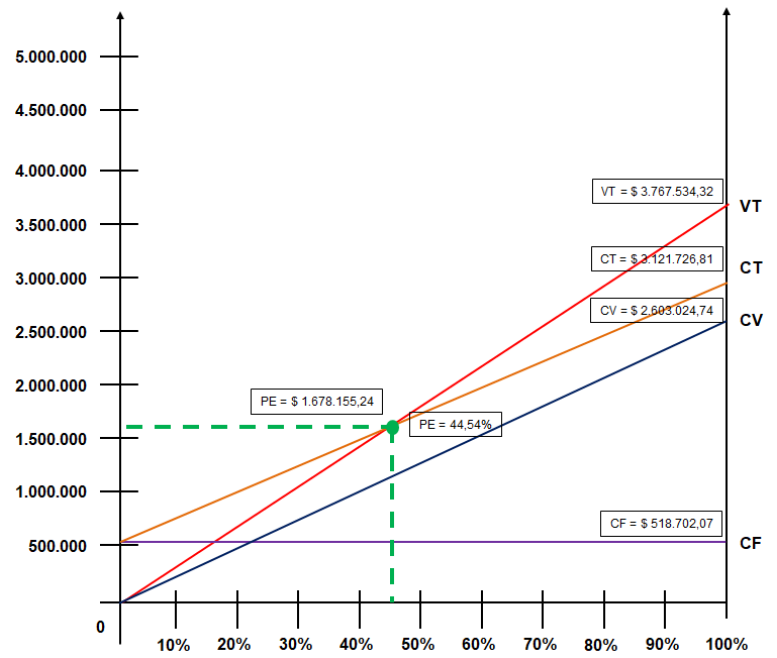

Figura 9. Punto de equilibrio

Fuente: Elaboración propia.

\section{Conclusiones.}

$>$ Los miembros de la Asociación San Antonio de Guayllabamba con los recursos que genera la producción actual de leche no han logrado mejorar su nivel social y económico, lo que ha evidenciado la necesidad de contar con estrategias de mejoramiento para la organización.

$>$ Quienes conforman la Asociación San Antonio de Guayllabamba mediante la investigación de pudo determinar la disposición de aquellas personas a capacitarse e implementar métodos en este caso la planta de enfriamiento obteniendo mejores réditos económicos, la comercialización, el desarrollo y bienestar de sus integrantes y sus familias.

$>$ Mediante la implementación de una planta de enfriamiento de leche las personas miembros de la Asociación San Antonio de Guayllabamba han visto necesaria la opción de mantener en pie el mercado de la leche dando respuesta a los requerimientos de sus contendientes y así dar mejor valor agregado al producto.

$>$ Se consideran necesario la implementación de la planta de enfriamiento de leche como una alternativa para fomentar la innovación tecnológica acorde a las exigencias competitivas del mercado actual debido a la carencia de asistencia técnica y financiera en la Asociación San Antonio de Guayllabamba lo que no les ha permitido consolidarse como una organización que trabaja de manera sostenible

$>$ La investigación ha podido evidenciar que los socios de la Asociación San Antonio de Guayllabamba requieren la implementación de una planta de enfriamiento en donde los productores de leche puedan dar un valor agregado a su producto, controlar las normas de 
calidad, salubridad y brindar la oportunidad a que todos oferten su producto respetando la política de precios por lo que están dispuestos a colaborar con sus recursos económicos y humanos para lograr la creación de la misma.

\section{Referencias bibliográficas}

AGSO. (2016). Financiamiento para la adquisición de ganado vacuno y otros activos destinados al mejoramiento de la producción lechera en la hacienda.

Assidon, E. (2002). Teorías económicas del desarrollo. Quito: Abya-Yala.

Baran, P. (1959). La economía política del crecimiento. México: Ed. segunda en español.

Barsky, O. (1982). Políticas agrarias, colonización y desarrollo rural en el Ecuador. Quito.

Baumann, B. (2004). Aplicación de estrategias de desarrollo enfocadas hacia las personas en el ámbito de la FAO. Obtenido de http://www.fao.org/docrep/007/j3137s/j3137s0a.htm

Bazoberry. (2010). Concepto, contexto y enfoques. En ¿Qué esperar de las ONG?: Enfoques y prácticas de desarrollo rural en los países andinos. Quito: Abya-Yala.

BCE. (2016). Banco Central del Ecuador; Producción lechera en la Comunidad Andina.

Bocco, M. (2007). Evaluación Socioeconómica de Sistemas Productivos Sustentables en el Chaco. Argentina: Producción Animal Zonas Áridas.

Bretón. (1999). Modelos “rotos” y modelos “por construir” en América Latina y África. Barcelona; Icara.

Bretón, V. (2002). Cooperación al desarrollo, capital social, y neoindigenismo en los Andes ecuatorianos. Revista europea de estudios Latinoamericanos y del Caribe.

Cánovas, F. (2000). La Mejora y gestión de los procesos del negocio”. Mejora y Gestión de Procesos. No. 9.

Cabra, C., \& Del Pilar, S. C. (2007). Diseño del sistema de calidad de recepción de leche cruda en Lácteos Simijaca SA Lacsimi SA Freskaleche SA en el municipio de Simijaca Cundinamarca.

Ceña, F. (1994). Planteamientos económicos del desarrollo rural: perspectiva histórica. Revista de estudios agro-sociales. Obtenido de http://helvia.uco.es/xmlui/bitstream/handle/10396/5705/rea.7.pdf?sequence=1 
Vol. 4, N¹., p.116-130 enero - marzo, 2020

Coraggio, J. (2016). La economía social y solidaria ante la pobreza. Obtenido de 2016:

http://www.vocesenelfenix.com/content/la-econom\%C3\%AD-social-y-solidaria-ante-lapobreza

Dairy. (2016). World markest and Trade.

Durango, T. (2013). Evaluación Socio-económica del uso de la pesca artesanal en cuatro comunidades Kichwa de la ribera del río Napo, Ecuador. Quito: USQ.

FAO. (2016). Mercado internacional del consumo de lácteos.

Hirschman, A. (1980). Auge y ocaso de la teoría económica del desarrollo. El trimestre económico,

http://www.jstor.org/stable/pdfplus/23395022.pdf?\&acceptTC=true\&jpdConfirm=true

INIAP. (2010). "Diagnóstico agro-socioeconómico de la actividad lechera en la provincia de Cotopaxi.

Montufar, E. (2014). Propuesta para el desarrollo económico de la población rural de la sierra centro del Ecuador a partir de su evaluación socioeconómica en el periodo 2001-2010 y mediante la economía popular y solidaria. Quito.

Pérez, J. (2014). Análisis Económico de la Producción de Leche Ecológica.

Revilla, A. (1982). Tecnología de la leche: procesamiento, manufactura y análisis (No. IICA LME 53). IICA, San José (Costa Rica).

Serrano, C. (2005). La política social en la globalización: programas de protección en América Latina (Vol. 70). United Nations Publications.

Torres, Y. (2013). Caracterización socioeconómica y productiva de las granjas de doble propósito orientadas a la producción de leche en una región tropical de Ecuador. Caso de la provincia de Manabí. Revista Científica, FCV-LUZ, Vol. XXV, N4.

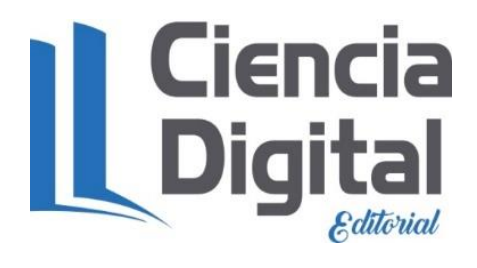


ISSN: 2602-8506

Vol. 4, $\mathrm{N}^{\circ} 1 .$, p.116-130 enero - marzo, 2020

\section{PARA CITAR EL ARTÍCULO INDEXADO}

Oleas Carrillo, E. R. O. C., Velasco Matveev, L. A. V. M., Benavides Lara, J. C., \& Burgos Mejía, M. A. (2020). Alternativa de crecimiento económico en el cantón Chambo mediante el proyecto de factibilidad de la planta de enfriamiento de leche. Visionario Digital, 4(1), 116-130. https://doi.org/10.33262/visionariodigital.v4i1.1067

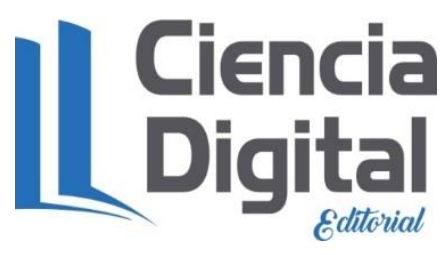

El artículo que se publica es de exclusiva responsabilidad de los autores y no necesariamente reflejan el pensamiento de la Revista Visionario Digital.

El artículo queda en propiedad de la revista y, por tanto, su publicación parcial y/o total en otro medio tiene que ser autorizado por el director de la Revista Visionario Digital.
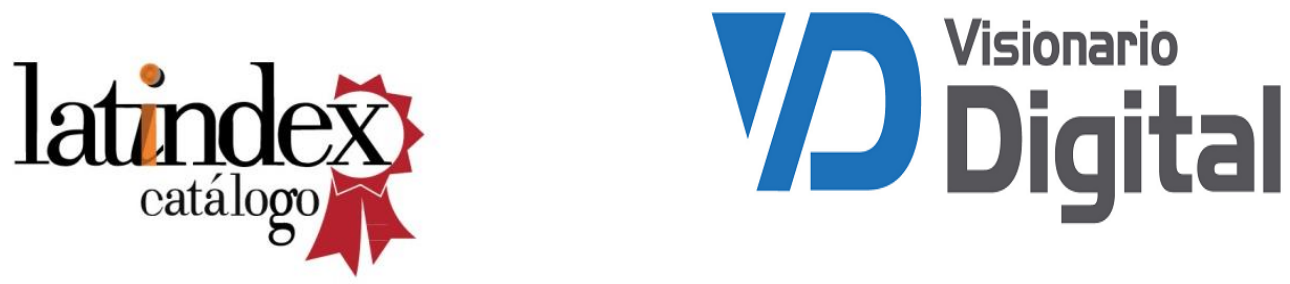